\title{
Trap losses induced by near-resonant Rydberg dressing of cold atomic gases
}

\author{
J. A. Aman, ${ }^{1}$ B. J. DeSalvo, ${ }^{1}$ F. B. Dunning, ${ }^{1}$ T. C. Killian, ${ }^{1}$ S. Yoshida, ${ }^{2}$ and J. Burgdörfer ${ }^{2}$ \\ ${ }^{1}$ Department of Physics and Astronomy, Rice University, Houston, Texas 77005-1892, USA \\ ${ }^{2}$ Institute for Theoretical Physics, Vienna University of Technology, Vienna, Austria, EU
}

(Received 13 March 2016; published 29 April 2016)

\begin{abstract}
The near-resonant dressing of cold strontium gases and Bose-Einstein condensates contained in an optical dipole trap (ODT) with the $5 s 30 s^{3} S_{1}$ Rydberg state is investigated as a function of the effective two-photon Rabi frequency, detuning, and dressing time. The measurements demonstrate that a rapid decrease in the ground-state atom population in the ODT occurs even for weak dressing and when well detuned from resonance. This decrease is attributed to Rydberg atom excitation, which can lead to direct escape from the trap and to population of very long-lived $5 s 5 p^{3} P_{0,2}$ metastable states. The effects of interactions between Rydberg atoms, including those populated by blackbody radiation, are analyzed. The work has important implications when considering the use of Rydberg dressing to control the interactions between dressed ground-state atoms.
\end{abstract}

DOI: 10.1103/PhysRevA.93.043425

\section{INTRODUCTION}

The study of cold quantum gases has led to many fundamental advances in understanding the behavior of interacting many-body systems, allowing, for example, the observation of the Bose-Einstein-condensate(BEC)-BCS crossover, the creation of Fermi superfluids, and the observation of the Mott insulator-superfluid transition in optical lattices. While these processes are driven by simple short-range contact interactions, much stronger interactions can be realized by taking advantage of long-range dipole-dipole interactions between Rydberg atoms, polar molecules, or atoms having large dipole moments. Theory predicts that such interactions can lead to a variety of novel phenomena, including phase transitions to strongly correlated classical crystals [1-4], roton-maxon excitations [5], three-dimensional solitons [6], and supersolids $[2,5,7,8]$.

Interactions between "ground-state" atoms can be controlled by the introduction of a small admixture of Rydberg character into their wave functions, with the strength of their interactions tuned by varying the size of the admixed fraction. Since Rydberg atoms experience strong long-range interactions, even a small admixture of Rydberg character can result in relatively strong atom-atom interactions. Such an admixture can be realized by dressing ground-state atoms with optical radiation tuned near resonance with the transition to a Rydberg state [9-11]. Here we explore the dressing of strontium atoms contained in a cold gas or BEC with the $5 s 30 s^{3} S_{1}$ Rydberg state, which introduces nearly isotropic repulsive interactions. This is accomplished using the twophoton excitation scheme illustrated in Fig. 1. Figure 1 also defines the relevant Rabi frequencies $\Omega_{g p}$ and $\Omega_{p r}$, detunings $\delta_{g p}$ and $\Delta$, and decay rates $\gamma_{p}$ and $\gamma_{r}$. For weak dressing (i.e., $\delta_{g p} \gg \Omega_{g p}$ and $\Delta \gg \Omega_{\mathrm{eff}}$, where $\Omega_{\mathrm{eff}}$ is the effective twophoton Rabi frequency $\left.\Omega_{g p} \Omega_{p r} / 2\left|\delta_{g p}\right|\right)$, the dressed ground state may be written as

$$
\left|g^{\prime}\right\rangle=\left(1-\frac{\Omega_{g p}^{2}}{8 \delta_{g p}^{2}}-\frac{\Omega_{\mathrm{eff}}^{2}}{8 \Delta^{2}}\right)|g\rangle+\frac{\Omega_{g p}}{2 \delta_{g p}}|p\rangle+\frac{\Omega_{\mathrm{eff}}}{2 \Delta}|r\rangle,
$$

where $|g\rangle,|p\rangle$, and $|r\rangle$ denote the undressed ground, intermediate $P$, and Rydberg states, respectively. For typical cold gas or BEC densities, interactions between Rydberg atoms dominate. Thus the interaction strength can be manipulated by varying $\Omega_{\text {eff }}$ and $\Delta$. Furthermore, whereas low- $\ell$ Rydberg states typically possess lifetimes of a few microseconds, dressed ground-state atoms with only a small admixture of Rydberg character have lifetimes on the order of milliseconds, suggesting longer coherence times. On the other hand, for high-density gases, even a small admixture of Rydberg character can result in strong interactions that involve multiple atoms. Such many-body effects can lead to deviations from the simple picture of isolated dressed atoms embodied in Eq. (1) and can introduce decoherence into the system [12].

Here we investigate the transition to the strong-interaction regime by dressing relatively dense clouds of thermal and Bose-condensed strontium atoms with peak densities $\rho$ of $\sim 4 \times 10^{11}$ and $\sim 5 \times 10^{13} \mathrm{~cm}^{-3}$, respectively, that are confined to an optical dipole trap. We observe that even for weak dressing and large overall detunings $\Delta$, the rate at which the ground-state atom population in the trap decreases is unexpectedly large. Such losses are important as they limit both the level and duration of the dressing that can be applied without incurring unacceptable trap losses. The processes responsible for trap loss are examined through a series of density- and time-dependent measurements. The data suggest that ground-state atom loss is associated with the excitation of Rydberg atoms, which can result in direct trap loss through recoil and in the population of very long-lived $5 s 5 p^{3} P_{0}$ and ${ }^{3} P_{2}$ metastable states, which also results in ground-state atom loss. Potential mechanisms that can enhance Rydberg atom production are discussed.

\section{EXPERIMENTAL TECHNIQUES}

Many aspects of the present apparatus are described in detail elsewhere $[13,14]$. Briefly, a sample of cold ${ }^{84} \mathrm{Sr}$ atoms is prepared in an optical dipole trap (ODT) formed using two crossed, focused, highly elliptical $1.06 \mu \mathrm{m}$ laser beams that generate a thin two-dimensional pancake-shaped trap with dimensions of $\sim 400 \times 300 \times 40 \mu \mathrm{m}^{3}$. Atoms loaded into the ODT are subject to forced evaporative cooling to create either cold thermal gas samples or BECs. The relevant trap frequencies and minimum trap depths are 9, 120, and $13 \mathrm{~Hz}$ and 


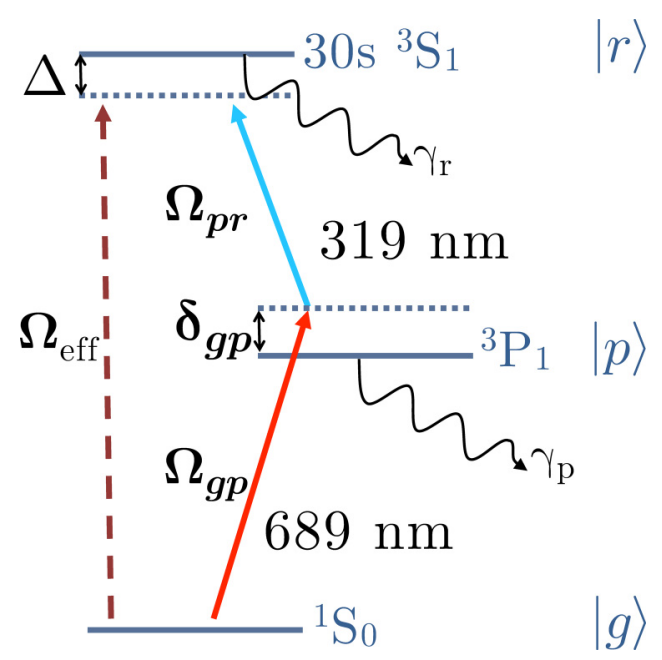

FIG. 1. Schematic of the dressing scheme employed showing the ${ }^{1} S_{0}$ ground state, the intermediate ${ }^{3} P_{1}$ state, and the ${ }^{3} S_{1}$ Rydberg state. The relevant Rabi frequencies $\Omega_{g p}$ and $\Omega_{p r}$, detunings $\delta_{g p}$ and $\Delta$, and decay rates $\gamma_{p}$ and $\gamma_{r}$ are indicated.

$0.84 \mu \mathrm{K}$, respectively, for the cold atomic gases and 8, 98, and $12 \mathrm{~Hz}$ and $0.32 \mu \mathrm{K}$ for the BECs. The estimated overall sample sizes for the cold gases and BECs are $\sim 150 \times 11 \times 103 \mu \mathrm{m}^{3}$ and $\sim 30 \times 2 \times 20 \mu \mathrm{m}^{3}$, respectively.

After preparation in the ODT, the atoms are dressed using the outputs of two lasers operating at 689 and $319 \mathrm{~nm}$ (see Fig. 1). Dressing with the $5 s 30 s^{3} S_{1}$ Rydberg state induces repulsive interactions between the ground-state atoms. A small magnetic bias field of 0.25 gauss is applied perpendicular to the plane of the trap and defines a quantization axis. The $689 \mathrm{~nm}$ beam travels parallel to the magnetic field and is circularly polarized. The $319 \mathrm{~nm}$ beam is incident perpendicular to the magnetic field and is linearly polarized parallel to the magnetic field. This configuration leads to the creation of $M_{J}=+1$ Rydberg states. The lateral dimensions of the beams are large compared to those of the trapped atom samples to ensure that all of the atoms are subject to the same uniform dressing. The data presented here were all recorded with the $689 \mathrm{~nm}$ laser blue detuned by $\delta_{g p}=2 \pi \times 40 \mathrm{MHz} . \Omega_{p r}$ was measured by Autler-Townes splitting [12] and set at $\Omega_{p r}=2 \pi \times 6 \mathrm{MHz} . \Omega_{g p}$ was determined with the aid of Rabi oscillations. Following dressing, the trap is maintained for a further $100 \mathrm{~ms}$ to allow time for recoiling atoms, which have velocities of $\sim 10^{-2} \mathrm{~ms}^{-1}$, to separate from the trap volume. The atoms remaining in the trap are then released and, after a fall time of $\sim 20 \mathrm{~ms}$, the number of ground-state atoms present is measured by absorption imaging on the ${ }^{1} S_{0} \rightarrow{ }^{1} P_{1}$ transition.

The recoil energy acquired by a ground-state atom upon excitation to the $30{ }^{3} S_{1}$ Rydberg state is equivalent to $\sim 1.3 \mu \mathrm{K}$. Once excited, $30{ }^{3} S_{1}$ Rydberg atoms decay with an effective lifetime of $1 / \gamma_{r} \sim 5 \mu \mathrm{s}$ [15] both through spontaneous decay to the $5 s 5 p^{3} P_{0,1,2}$ states and through blackbody-radiation (BBR)-induced transitions to neighboring high- $n P$ states. While the lifetime of the $5 s 5 p^{3} P_{1}$ state against decay back to the ${ }^{1} S_{0}$ ground state is relatively short $\left(1 / \gamma_{p} \sim 21.5 \mu \mathrm{s}\right)$, the Wigner-Eckart theorem for the transition matrix suggests that in the absence of spin-dependent interactions, two-thirds of decays to $5^{3} \mathrm{P}$ levels will result in the production of ${ }^{3} P_{0}$ and ${ }^{3} P_{2}$ states whose lifetimes are much longer than the time scale of the present experiments. While the ${ }^{3} P_{0}$ and ${ }^{3} P_{2}$ states may remain trapped in the ODT, they are not imaged and their production therefore constitutes a loss of ground-state atoms from the trap. Decay of the $5^{3} P_{1}$ states results in further energy transfer to or from the atoms, broadening their energy distribution. In the case of a BEC, following decay, the great majority of the product ground-state atoms will have more than enough energy to escape the ODT. Although the ODT used to contain the cold gases is somewhat deeper, a large fraction of the product atoms is still able to escape. Also, at high excited-state densities, energy transfer resulting from collisions can become important and enhance trap loss. Rydberg atom excitation, therefore, can instigate a variety of processes that deplete the ground-state atom population in the trap, suggesting it is the primary driver for the observed trap loss. The resulting loss is measured as a function of Rabi frequency $\Omega_{\text {eff }}$, detuning $\Delta$, and exposure time.

\section{RESULTS AND DISCUSSION}

Initial experiments were undertaken using cold, nondegenerate gases containing $\sim 5 \times 10^{5}$ atoms with peak densities of $\sim 4 \times 10^{11} \mathrm{~cm}^{-3}$. Surprisingly, large ground-state atom losses were observed even when well detuned from resonance where the dressing is weak. This is illustrated in Fig. 2, which shows the ground-state atom losses seen for Rabi frequencies $\Omega_{\mathrm{eff}}$ in the range $2 \pi \times 35$ to $2 \pi \times 200 \mathrm{kHz}$. The dressing times $t_{D}$ were varied from $\sim 200$ to $800 \mu \mathrm{s}$ and were chosen to maintain similar ground-state atom losses on resonance. For the smallest value of $\Omega_{\text {eff }}$, the loss feature can be fit well by a Gaussian distribution. As $\Omega_{\text {eff }}$ is increased, the loss features broaden and become noticeably asymmetric, with somewhat larger losses being apparent for blue detuning. For the largest value of $\Omega_{\text {eff }}$, even for a red detuning $\Delta \sim 2 \pi \times 9 \mathrm{MHz}$, which corresponds to a Rydberg occupation probability in the dressed state [Eq. (1)], $\sigma_{0}=\Omega_{\text {eff }}^{2} / 4 \Delta^{2} \sim 1.2 \times 10^{-4}$, approximately one-third of the ground-state atoms initially present in the trap are lost after dressing for only $t_{D} \sim 200 \mu \mathrm{s}$.

The effects of increased sample density (and smaller trap depth) are seen in Fig. 3, which presents data obtained using a BEC containing $\sim 5 \times 10^{5}$ atoms with a peak density of $\sim 5 \times 10^{13} \mathrm{~cm}^{-3}$ and the same values of $\Omega_{\text {eff }}$ and $t_{D}$ as in Fig. 2. Comparison of Figs. 2 and 3 shows that increases in sample density lead to further increases in the widths of the ground-state atom loss features. As $\Omega_{\text {eff }}$ increases, the peaks again become noticeably asymmetric. For the largest value of $\Omega_{\text {eff }}$, a one-third depletion in the number of ground-state atoms present is seen after $200 \mu \mathrm{s}$ for a detuning $\Delta \sim 2 \pi \times 48 \mathrm{MHz}$. Note, however, that for such large detunings, $\Delta>\delta_{g p}$, the model of an effective two-level system is no longer adequate, with the corresponding eigenstate of the Hamiltonian having a larger contribution from the $5 s 5 p^{3} P_{1}$ state.

The results in Figs. 2 and 3 demonstrate that even far off resonance, Rydberg dressing can lead to a rapid loss of ground-state atoms from the trap. To test if these losses were associated with the creation of charged particles, data similar to those in Figs. 2 and 3 were recorded with transverse electric 


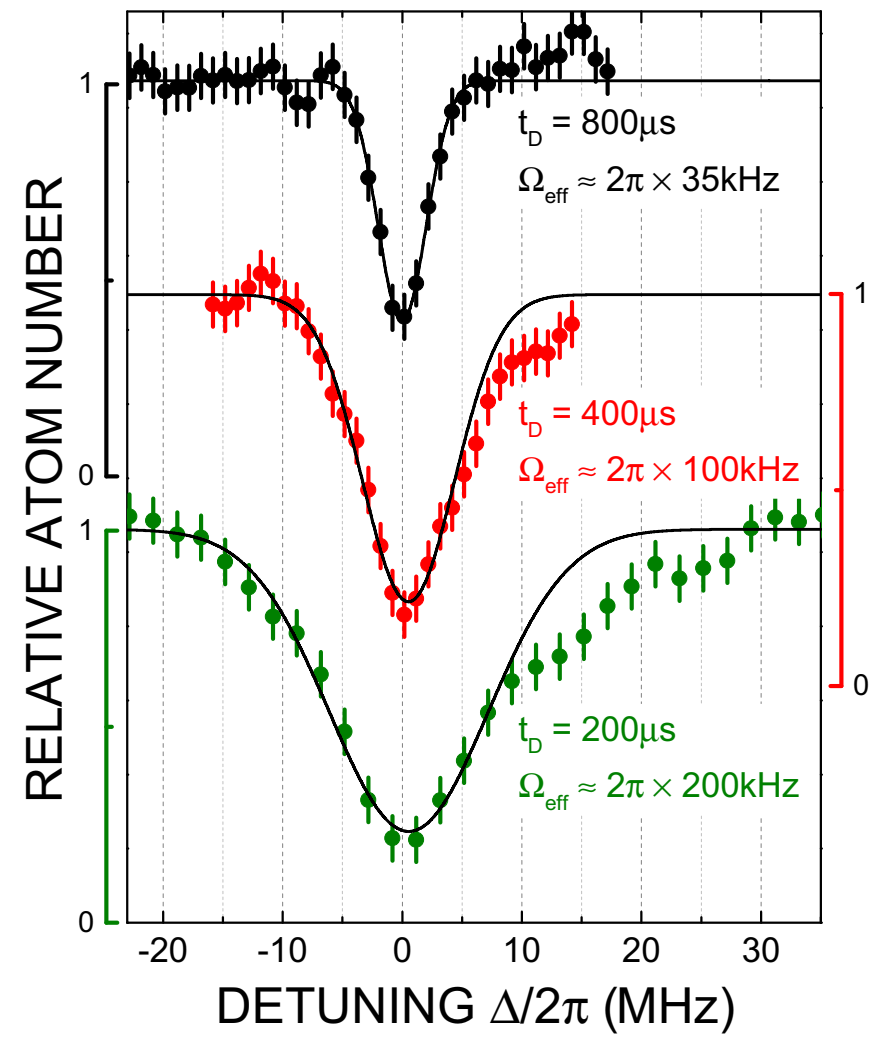

FIG. 2. Measured decrease in the number of ground-state atoms present in a cold strontium atom gas dressed with the $30{ }^{3} S_{1}$ Rydberg state as a function of detuning, $\Delta / 2 \pi$. The gas contains $\sim 5 \times 10^{5}$ atoms with a peak density $\sim 4 \times 10^{11} \mathrm{~cm}^{-3}$ and temperature $150 \mathrm{nK}$. The estimated Rabi frequencies $\Omega_{\text {eff }}$ and the dressing times $t_{D}$ used to obtain the different data sets are as indicated. The solid lines are drawn to guide the eye and represent Gaussian fits to the data recorded at red detunings.

fields of up to $1 \mathrm{~V} \mathrm{~cm}^{-1}$ applied across the trap volume using annular electrodes positioned outside two opposing windows. Such fields are sufficient to transport ions out of the volume occupied by the trapped atoms on time scales of $\sim 200 \mathrm{~ns}$ and $500 \mathrm{~ns}$ for the BECs and thermal samples, respectively. Both time scales are much less than the present dressing periods. Measurements, however, showed that application of such fields resulted in no discernible changes in the trap loss features, indicating that any effects associated with ion production must be small and not responsible for the present observations. The observed effects also cannot be attributed to the formation of long-range $\mathrm{Sr}_{2}$ Rydberg molecules in which scattering of the Rydberg electron from a neighboring ground-state atom binds the two together. While ancillary measurements confirmed the production of such species, their formation rates are much too small to account for the present observed trap losses [16].

The sizable increases in the widths of the trap loss features seen when the trap density is increased suggest that atomic spacings, as well as $\Omega_{\text {eff }}$, play an important role in trap loss. The probability distribution function that describes the internuclear separation $R$ to the nearest neighbor is given by

$$
P(R)=\frac{3}{a}\left(\frac{R}{a}\right)^{2} e^{-\left(\frac{R}{a}\right)^{3}},
$$

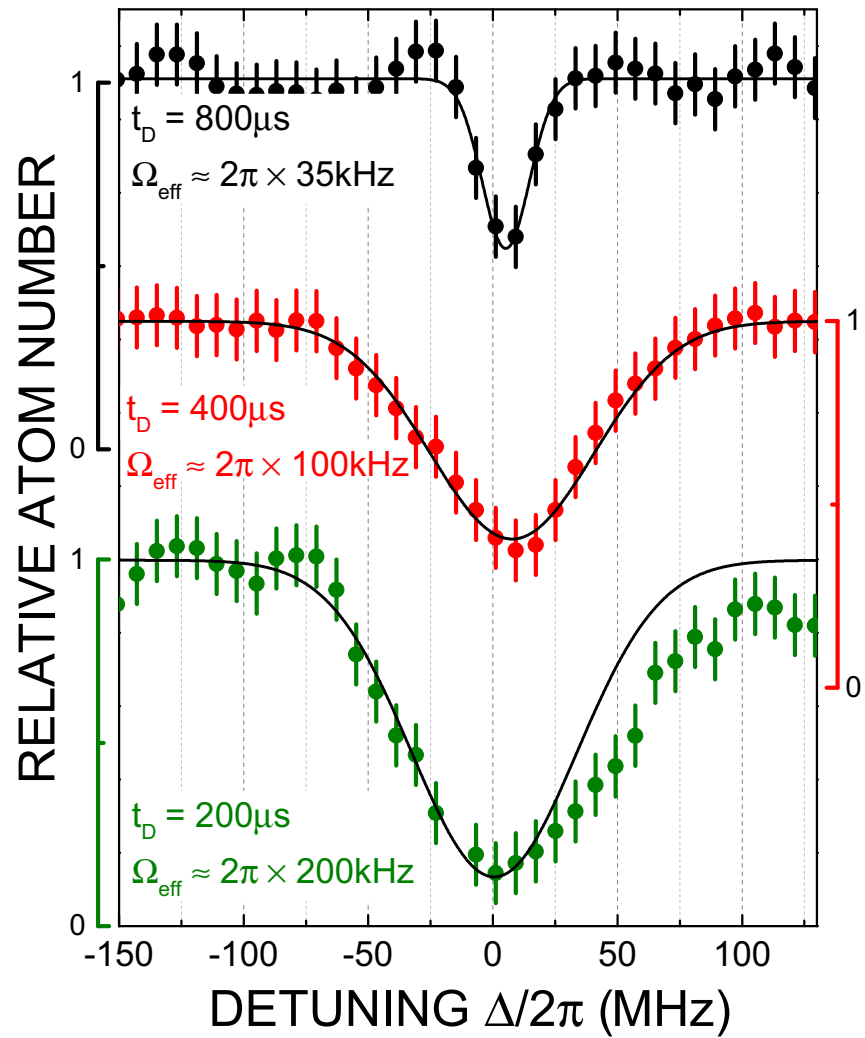

FIG. 3. Measured decrease in the number of ground-state atoms present in a BEC contained in the ODT and dressed with the $30{ }^{3} S_{1}$ Rydberg state as a function of detuning, $\Delta / 2 \pi$. The BEC contains $\sim 5 \times 10^{5}$ atoms with a peak density of $\sim 5 \times 10^{13} \mathrm{~cm}^{-3}$ and temperature of $\sim 30 \mathrm{nK}$. The estimated Rabi frequencies and the dressing times are the same as for Fig. 2. The solid lines show Gaussian fits to the data at red detunings.

where $a=(3 / 4 \pi \rho)^{1 / 3}$ is the Wigner-Seitz radius and $\rho$ is the local density. This distribution peaks at $R \sim 0.87 a$. Although the density will vary across the trap leading to a range of most-probable nearest-neighbor separations, typical average values are $a \sim 1.2 \mu \mathrm{m}$ for the cold gases and $a \sim 0.23 \mu \mathrm{m}$ for the BECs. The interaction strength for a pair of $30{ }^{3} S_{1}$ Rydberg atoms can be calculated as a function of $R$ using multipole expansion, including up to quadrupole-quadrupole interactions, and is shown in Fig. 4(a). (Note that the contributions beyond dipole-dipole interaction become visible only for small internuclear distance $R<500 \mathrm{~nm}$.) The blockade radius, which for a laser linewidth of $\sim 1 \mathrm{MHz}$ is $\sim 1.5 \mu \mathrm{m}$, is larger than the nearest-neighbor separation for both the thermal gas and the BEC. Therefore, it is not unreasonable to expect that interactions might influence the atom loss.

To gain further insight into the loss mechanism(s), a series of time-dependent measurements were undertaken using the BEC. When the interaction time is much longer than the lifetime $(\sim 5 \mu \mathrm{s})$ of the ${ }^{3} S_{1}$ Rydberg state, the measured atom loss is very likely dominated by population of the metastable ${ }^{3} P_{0,2}$ states whose time development is approximated by $N \sigma_{r}(2 / 3) \gamma_{r} t$, with $N \sigma_{r}$ being the number of Rydberg atoms and $(2 / 3) \gamma_{r}$ the decay rate of the ${ }^{3} S_{1}$ Rydberg state to 

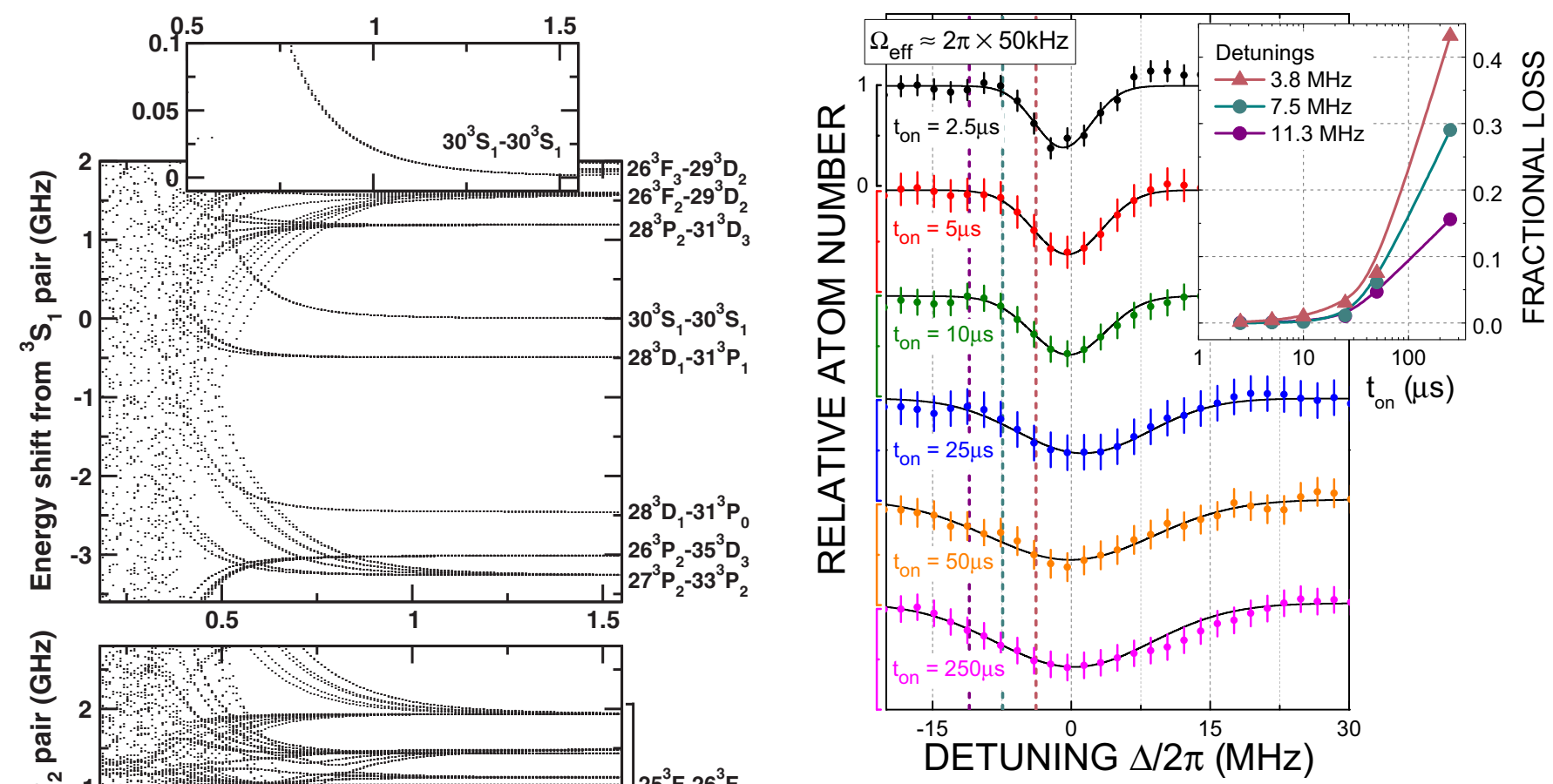

FIG. 5. Measured decrease in the relative number of ground-state atoms present in a dressed BEC as a function of detuning. The initial atom number, density, and temperature are as in Fig. 3. The estimated Rabi frequency $\Omega_{\text {eff }}$ is $\sim 2 \pi \times 50 \mathrm{kHz}$. Dressing is provided in a series of $N$ pulses, each of equal width, $t_{\mathrm{on}}$, and separated by $100 \mu \mathrm{s}$, keeping the total dressing $N \times t_{\text {on }}$ constant. The values of $N$ and $t_{\text {on }}$ employed to obtain the various data sets are as indicated. The solid lines show Gaussian fits to the data for red laser detunings. The inset shows the trap losses seen per pulse for red detunings of $\sim 0.8,7.5$, and $11.3 \mathrm{MHz}$ as a function of pulse width (see text). The lines are drawn to guide the eye.

FIG. 4. Calculated energy levels for (a) a $30{ }^{3} S_{1}-30{ }^{3} S_{1}$ Rydberg atom pair and (b) a $30{ }^{3} S_{1}-29{ }^{3} P_{2}$ Rydberg atom pair as a function of internuclear separation $R$. The inset shows the energy levels for a $30{ }^{3} S_{1}-30{ }^{3} S_{1}$ atom pair on an expanded scale. The calculations include the dipole-dipole and dipole-quadrupole interactions. Quadrupolequadrupole interactions are included only among degenerate states. The two atoms are aligned along the $z$ axis and the sum of the $M_{J}$ values for both atoms is set to zero.

the metastable $P$ states. Many-body effects can be seen as deviations of the Rydberg occupation probability $\sigma_{r}$ from the value expected, $\sigma_{0}=\Omega_{\text {eff }}^{2} /\left(4 \Delta^{2}\right)$, for an isolated dressed state. In particular, a reaction process in which creation of one, or a few, Rydberg atoms triggers further, more rapid Rydberg atom production can result in accelerated trap loss [i.e., $\sigma_{r}>\Omega_{\text {eff }}^{2} /\left(4 \Delta^{2}\right)$ ]. Similarly, at large detunings, such accelerated trap loss can lead to a broadening of the trap loss spectra beyond that expected from the line shape, $\Omega_{\text {eff }}^{2} /\left(2 \Omega_{\text {eff }}^{2}+\gamma_{r}^{2}+4 \Delta^{2}\right)$, for an isolated atom. Figure 5 shows trap loss data recorded with the dressing delivered in a series of $N$ pulses of equal width $t_{\text {on }}$ chosen such that the total dressing, i.e., $N t_{\text {on }}$, was constant. The pulses are each separated by $100 \mu \mathrm{s}$ which, because the Rydberg atoms decay with a lifetime of $\sim 5 \mu \mathrm{s}$ [15], is more than sufficient to allow Rydberg atoms produced during one pulse to decay prior to the next pulse. Application of a single $250 \mu$ s pulse leads to a broad

trap loss feature. The widths of the loss features, however, decrease as the dressing is divided into ever smaller segments. In the limit that $1002.5-\mu$ s dressing pulses are applied, the width of the loss feature is dramatically reduced, despite the fact that the laser parameters, i.e., $\Omega_{\text {eff }}$ and $\Delta$, and the total dressing are maintained constant. Such behavior provides an indication that interaction effects cause a broadening that only sets in at longer dressing times. The inset in Fig. 5 shows the trap loss seen per pulse for red detunings of $\sim 3.8,7.5$, and $11.3 \mathrm{MHz}$ as a function of $t_{\mathrm{on}}$. Little trap loss is evident for small values of $t_{\text {on }}$ and only begins to build up for values of $t_{\text {on }}$ greater than a few tens of microseconds. Once trap loss begins, however, it grows rapidly, consistent with an accelerating loss process.

As a further test, measurements were undertaken using a series of $2501-\mu$ s-long dressing pulses with a constant time separation $t_{s}$ between each pulse. Figure 6 shows trap loss data recorded for selected values of $t_{s}$ in the range 1 to $100 \mu \mathrm{s}$. As is evident from Fig. 6, even though the total dressing for each data set is similar, the width of the trap loss feature decreases markedly as the time delay between the pulses is increased, with the bulk of this decrease occurring as the interpulse spacing is increased from 1 to $10 \mu \mathrm{s}$. Because the majority of Rydberg atoms decays within $10 \mu \mathrm{s}$, the use of a 


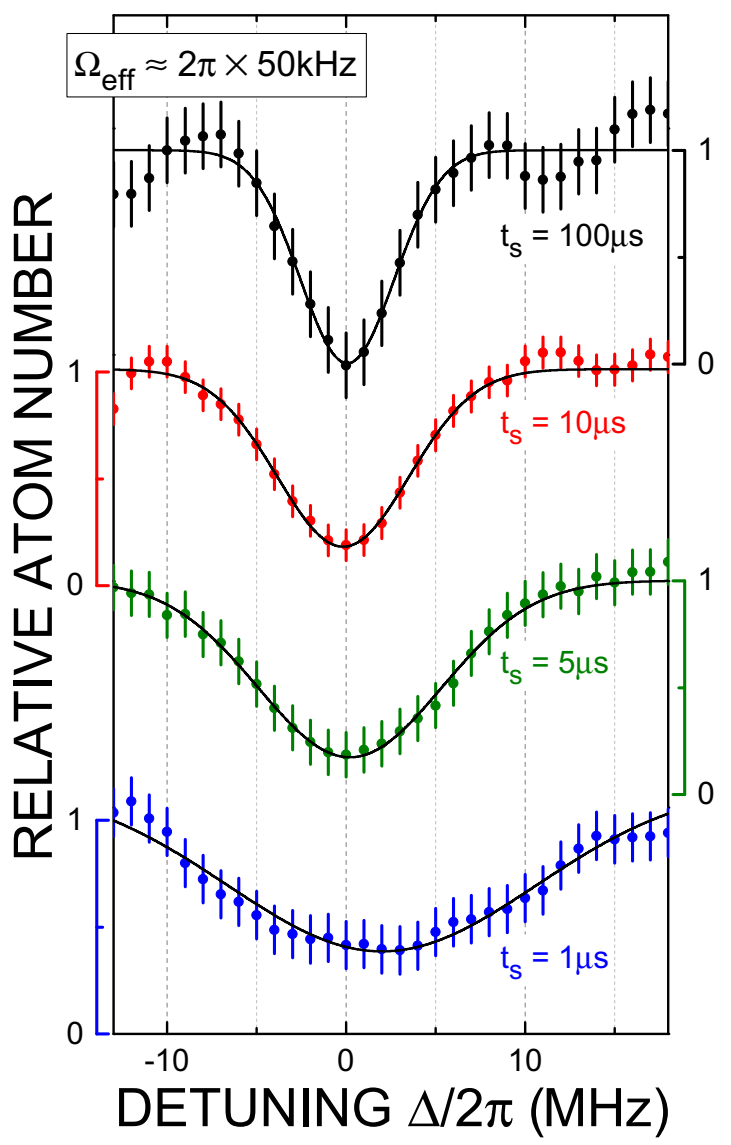

FIG. 6. Measured decrease in the relative number of ground-state atoms present in a dressed BEC as a function of detuning. The initial atom number, density, and temperature are as in Fig. 3. The dressing is provided by a series of $2501-\mu$ s-long pulses whose time separations $t_{s}$ are as indicated. The estimated Rabi frequency $\Omega_{\text {eff }}$ is $\sim 2 \pi \times$ $50 \mathrm{kHz}$. The curves show Gaussian fits to the data.

time separation $\gtrsim 10 \mu$ s between pulses allows most Rydberg atoms present in the condensate to decay prior to the next dressing pulse, which inhibits the growth of large numbers of Rydberg atoms.

The data in Figs. 5 and 6 point to ground-state atom losses from the trap that are triggered by the initial production of a few Rydberg atoms. The question then arises as to the physical processes that might account for this behavior. One mechanism that might lead to accelerated Rydberg atom production is that suggested by earlier workers studying the excitation of (repulsively interacting) Rydberg atoms in cold quasi-one-dimensional (quasi-1D) gases $[15,17]$ and in thin vapor cells [18]. Studies with quasi-1D gases revealed broad asymmetric excitation spectra together with increased secondand third-order statistical moments of the Rydberg number distribution. This behavior was attributed to a multistep process in which initial (off-resonant) creation of "seed" Rydberg atoms enabled the resonant excitation of neighboring atoms, facilitating the growth of localized groups of Rydberg atoms even when using radiation well (blue) detuned from resonance. Although the probability for creating seed Rydberg atoms is small at large detunings, the rate for their creation is, nonetheless, significant because of the large number of ground-state atoms present. Once created, seed Rydberg atoms can augment Rydberg atom production through resonant excitation and through decoherence of the dressed state [19], leading to the creation of Rydberg aggregates. Figure 4(a) shows the energy levels for strontium Rydberg atom pairs with values of $n$ near 30 as a function of internuclear separation $R$. Sizable shifts in the energies of the $30{ }^{3} S_{1}$ pair states are evident. They increase as $R$ decreases and are $\sim 20 \mathrm{MHz}$ for values of $R \sim 1 \mu \mathrm{m}$. Thus a ground-state atom positioned at an appropriate distance from a $30{ }^{3} S_{1}$ Rydberg atom can, with blue laser detuning, be resonantly excited to the same Rydberg state. Such excitation can then trigger resonant excitation of other neighboring atoms, leading to creation of a rapidly expanding cloud of Rydberg atoms. Nonetheless, it seems unlikely that this is the dominant process responsible for the present observations because, while it can account for the enhanced trap loss seen when blue detuned, it cannot explain the large trap losses seen when red detuned. Additionally, unlike rubidium, the ${ }^{3} S_{1}$ Rydberg state can decay to metastable ${ }^{3} P_{0,2}$ states, which steadily depletes the ground-state atom population. Creation of $30{ }^{3} S_{1}$ Rydberg atoms, however, can also lead to population of neighboring high- $n, n^{3} P_{0,1,2}$ states through BBR-induced transitions. As shown by the calculations in Fig. 4(b), $29^{3} P_{2}$ atoms interact strongly with $30{ }^{3} S_{1}$ atoms, resulting in the appearance of both red- and blue-shifted pair states whose energy shifts are, for a given internuclear separation, much larger than for a $30{ }^{3} S_{1}-30{ }^{3} S_{1}$ atom pair. Such shifts allow ground-state atoms positioned near a $29^{3} P_{J}$ atom to be resonantly excited to the $30{ }^{3} S_{1}$ Rydberg level for both red and blue laser detuning. The presence of even a few $29^{3} P_{J}$ atoms can have a major impact on Rydberg production because, for detunings of $20 \mathrm{MHz}$, resonance can be achieved for various values of internuclear separation up to $R \sim 2.4 \mu \mathrm{m}$. This strong, nonisotropic interaction increases the probability for interaction-induced resonant Rydberg excitation well above that expected for excitation in the presence of a ${ }^{3} S_{1}$ atom. In addition, calculations show that the $29{ }^{3} P_{0,1,2}$ states have long effective lifetimes, $\sim 30 \mu \mathrm{s}$, and that their decay is governed primarily by BBR-induced transitions to neighboring Rydberg states which can also interact strongly with the $30{ }^{3} S_{1}$ state.

To gauge the importance of Rydberg ${ }^{3} P_{0,1,2}$ states in influencing trap loss, a series of coupled many-body rate equations was solved using a Monte Carlo method. The use of rate equations to examine trap loss is reasonable if, as postulated here, the creation of seed Rydberg atoms can lead to decoherence of the dressed state that further enhances Rydberg atom production. To simulate as many atoms as possible, the rate equations are simplified by assuming a constant dephasing rate and by including only the time evolution of the occupation probabilities for the ground state $\sigma_{g, i}$, the ${ }^{3} S_{1}$ Rydberg state $\sigma_{r, i}$, the ${ }^{3} P_{J}$ Rydberg states $\sigma_{\mathrm{P} \text { Ryd }, i}$, and the metastable $5{ }^{3} P_{0,2}$ states, $\sigma_{m, i}$, where $i$ denotes the $i$ th atom $(i=1,2, \ldots, N)$. The rates for spontaneous decay of the Rydberg state to the ground state (via the $5{ }^{3} P_{1}$ metastable state), $\gamma_{r-g}=(1 / 3) \gamma_{r}=$ $6.7 \times 10^{4} \mathrm{~s}^{-1}$, and to the $5^{3} P_{0,2}$ metastable states, $\gamma_{r-m}=$ $(2 / 3) \gamma_{r}=1.3 \times 10^{5} \mathrm{~s}^{-1}$, were calculated together with the BBR-induced transition rate $\gamma_{\mathrm{BB}}=5.0 \times 10^{4} \mathrm{~s}^{-1}$ between the $S$ and $P$ Rydberg states. The coupled rate equations can be 
written as

$$
\begin{aligned}
\frac{d}{d t} \sigma_{g, i}= & -W_{i} \sigma_{g, i}+\left(W_{i}+\gamma_{r-g}\right) \sigma_{r, i}, \\
\frac{d}{d t} \sigma_{r, i}= & W_{i} \sigma_{g, i}-\left(W_{i}+\gamma_{r-g}+\gamma_{r-m}+\gamma_{\mathrm{BB}}\right) \sigma_{r, i} \\
& +\gamma_{\mathrm{BB}} \sigma_{\mathrm{PRyd}, i}, \\
\frac{d}{d t} \sigma_{\mathrm{P} \mathrm{Ryd}, i}= & \gamma_{\mathrm{BB}} \sigma_{r, i}-\gamma_{\mathrm{BB}} \sigma_{\mathrm{P} \mathrm{Ryd}, i}, \\
\frac{d}{d t} \sigma_{m, i}= & \gamma_{r-m} \sigma_{r, i} .
\end{aligned}
$$

The equations are coupled via the interaction $V_{\text {int }, i}=$ $\sum_{j \neq i} V_{i j}$, which modifies the excitation rate $W_{i}$. For noninteracting atoms $\left(V_{\text {int }}=0\right)$, the stationary solution matches the corresponding single-atom Lindblad equation when the excitation rate is set to

$$
W_{i}=W_{i}\left(V_{\mathrm{int}}=0\right)=\frac{\Omega_{\mathrm{eff}}^{2}\left(\gamma_{r-g}+\gamma\right)}{\left(\gamma_{r-g}+\gamma\right)^{2}+4 \Delta^{2}},
$$

where $\gamma$ is the dephasing rate for the ${ }^{3} S_{1}$ Rydberg state.

In the Monte Carlo simulations [20], the positions of $N$ atoms are randomly distributed according to a Gaussian distribution mimicking the density distribution in the BEC. The effective size of the sample increases with $N$ to maintain the average density. During the time evolution, the positions of the atoms are assumed to be fixed. Initially all atoms are in the ground state and the states of atoms, $\left\{\alpha_{1}(k), \alpha_{2}(k), \ldots, \alpha_{N}(k)\right\}\left[\alpha_{i}(k)=g, r, \mathrm{P}\right.$ Ryd or $\left.m\right]$, are updated stochastically at every time step, $t=k \Delta t(k=$ $1,2,3, \ldots)$, using random numbers $r$ generated uniformly in [0,1]. For example, when atom $i$ is in state $\xi$ at $t=k \Delta t$ [i.e., $\alpha_{i}(k)=\xi$ ] and the transition probability to a state $\eta$ is given by $T_{\xi \eta}$, the state of the atom is updated to $\alpha_{i}(k+1)=\eta$ only when $r<T_{\xi \eta}$. According to the rate equations [Eqs. (3)], the transition probabilities are given by $\gamma_{\beta} \Delta t(\beta=r-g, r-$ $m, \mathrm{BB})$ or $W_{i} \Delta t$ for small $\Delta t$. The time development of atom loss probability can be evaluated by counting the number of ground-state atoms in each time step. By averaging over many realizations of the stochastic processes, the statistical error can be reduced and the final results converge to the solution of the rate equations. Additionally, by regenerating the positions of atoms at each realization, the convolution with the Gaussian spatial distribution is properly taken into account. This stochastic modeling of electronic state dynamics is known to be applicable for stochastic processes such as spontaneous decay and BBR-induced transitions [21]. Laser excitation is a coherent and deterministic process, but the collapse of the excited dressed state due to decoherence adds stochasticity and the Monte Carlo approach becomes applicable.

To account for the correlated dynamics of $N$ atoms due to interactions, the states of the $N$ atoms within a single time step are updated in the following way. At each time step, a random ordering of the $N$ atoms, $\left\{i_{1}, i_{2}, \ldots, i_{N}\right\}$, is prepared, and the determination of whether each atom undergoes a transition is made as described above. The changes in $\alpha_{i}(k)$ do not affect the rates for spontaneous decay and BBR-induced processes. However, the transition rates for laser (de)excitation $W_{i}$ depend upon the state of all atoms $\alpha_{j}(k)(j \neq i)$, as described below.
Once the state of atom $i_{a}$ is updated, the transition rate $W_{i_{b}}$ is reevaluated for subsequent atoms $(b>a)$. Since each time step and each realization chooses a different random permutation, the final results do not bias any specific sequential process.

The effects of many-body interactions on the rates $W_{i}$, such as the dipole blockade, are treated by shifting the energy of the ${ }^{3} S_{1}$ Rydberg state due to all pairwise interactions, $\Delta \rightarrow \Delta-V_{\text {int }, i}$, where $V_{\text {int }, i}=\sum_{j \neq i} V_{i j}$. For the sum, the atom $i$ is always assumed to be in the ${ }^{3} S_{1}$ Rydberg state. On the other hand, the state of atom $j$ is taken from the Monte Carlo simulation. When the atom $j$ is in either the ground or the metastable $5{ }^{3} P_{0,2}$ state, the interaction is negligibly small, $V_{i j}=0$. If atom $j$ is in the ${ }^{3} S_{1}$ Rydberg state, the two-body interaction $V_{i j}$ is given by $C_{6} / R_{i j}^{6}$, and $C_{6}=2 \pi \times 21 \mathrm{MHz} \mu \mathrm{m}^{6}$ is obtained from a fit to Fig. 4(a). When atom $j$ is in the ${ }^{3} P_{J}$ Rydberg state, the situation is more complicated. In the current model, several ${ }^{3} P_{J}$ Rydberg states with different values of $n$ and $J$ are represented by a single state. Additionally, for a given $n$ and $J$, there are multiple nondegenerate eigenstates for a pair of ${ }^{3} S_{1}$ and ${ }^{3} P_{J}$ Rydberg states, and the energy shifts of the eigenstates depend also on the sum $M_{J, i}+M_{J, j}$ quantized along the alignment of two atoms. Therefore, the exact interaction energy is difficult to determine. Summing over multiple $P$ state atoms complicates the energy structure even further as the oscillator strength depends on the alignment of the two atoms relative to the laser polarizations. To simplify the situation, the oscillator strengths and the energy levels are continuously and uniformly distributed in the range $\left[-V_{\max , i}^{P}, V_{\max , i}^{P}\right]$, with $V_{\max , i}^{P}=\sum_{\alpha_{j}=P} C_{3} / R_{i j}^{3} . C_{3}=2 \pi \times 291 \mathrm{MHz} \mu \mathrm{m}^{3}$ is obtained from a fit to the eigenstate with the maximum energy shift connecting to the $S+P$ asymptote in Fig. 4(b). Integrating over all eigenstates, the excitation rate becomes

$$
\begin{aligned}
W_{i}= & \frac{1}{2 V_{\max , i}^{P}} \\
& \times \int_{-V_{\max , i}^{P}}^{V_{\max , i}^{P}} \frac{\Omega_{\mathrm{eff}}^{2}\left(\gamma_{r-g}+\gamma\right)}{\left(\gamma_{r-g}+\gamma\right)^{2}+4\left(\Delta-V_{\mathrm{int}, i}^{S}-V_{\mathrm{int}, i}^{P}\right)^{2}} d V_{\mathrm{int}, i}^{P},
\end{aligned}
$$

where $V_{\mathrm{int}, i}^{S}=\sum_{\alpha_{j}=R} C_{6} / R_{i j}^{6}$ is the interaction energy of atom $i$ due to surrounding atoms in the ${ }^{3} S_{1}$ Rydberg state.

Using the Monte Carlo approach, systems with up to $N=100000$ atoms are simulated. The (ground-state) atom loss probability at a given time is estimated by $\sigma_{\text {loss }}=$ $N^{-1} \sum_{i}\left(\sigma_{r, i}+\sigma_{\mathrm{PRyd}, i}+\sigma_{m, i}\right)$. Figure $7(\mathrm{a})$ shows the atom loss probability as a function of time for several different values of $N$. At large detuning [Fig. 7(a)], atom loss initially increases rapidly with $N$, but appears to saturate for $N>50000$, which corresponds to a sample whose largest dimension is $R \sim 10 \mu \mathrm{m}$ (i.e., $C_{3} / R^{3} \sim 2 \pi \times 0.3 \mathrm{MHz}$ ). Moreover, the simulations show that without BBR-induced transitions to the Rydberg ${ }^{3} P_{J}$ states (i.e., with $\gamma_{\mathrm{BB}}=0$ ), the atom loss probability does not grow significantly with increasing $N$, which indicates that it is the interaction between the ${ }^{3} S_{1}$ and ${ }^{3} P_{J}$ Rydberg states that is responsible for the acceleration in the atom loss rate. 


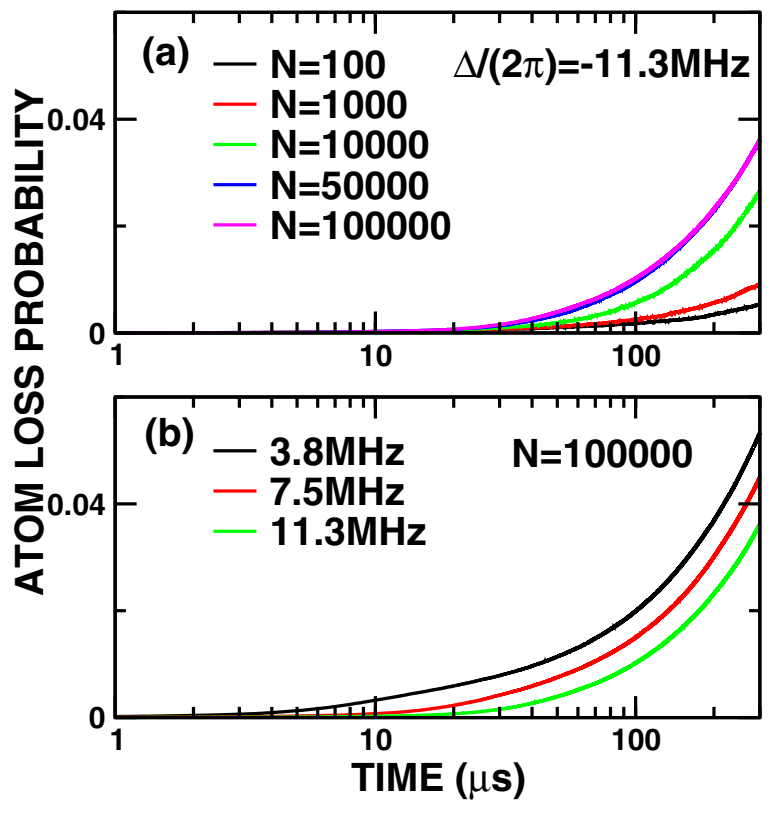

FIG. 7. Calculated time dependence of the atom loss probability $\sigma_{\text {loss }}(t)$ (see text). (a) The (red) detuning is set to $\Delta=-2 \pi \times$ $11.3 \mathrm{MHz}$ and the number $N$ of atoms is as indicated. (b) $N=$ 100000 and the detunings $\Delta$ are as indicated. The dephasing rate $\gamma$ is set to $5 \times 10^{6} \mathrm{~s}^{-1}$.

Figure 7(b) shows predictions of trap loss made using $N=100000$ atoms and the same detunings as in Fig. 5. The results are in reasonable qualitative agreement with the experimental observations. In particular, the initial trap loss is less for the larger detunings due to the slower creation of seed Rydberg atoms, which slows the production of the ${ }^{3} P_{J}$ Rydberg atoms. With only a few ${ }^{3} P_{J}$ Rydberg atoms present, many ground-state atoms remain well separated from such atoms, resulting in a small interaction strength $V_{\max , i}^{P} \ll$ $\left|\Delta-V_{\mathrm{int}, i}^{S}\right|$. In this limit, the excitation rate $W_{i}$ [Eq. (5)] increases as more ${ }^{3} P_{J}$ Rydberg atoms are populated and $V_{\mathrm{int}, i}^{P}$ grows. At late times, however, when $V_{\mathrm{int}, i}^{P}$ reaches the limit $V_{\max , i}^{P} \gg\left|\Delta-V_{\text {int }, i}^{S}\right|$, the rate $W_{i}$ becomes dependent on $V_{\max , i}^{P}$ and rather insensitive to detuning $\Delta$. This is seen in
Fig. 7(b), where the results show a clear dependence on $\Delta$. This is partly because the interaction strength $V_{\max , i}^{P}$ becomes much larger than the detuning and small differences in $\Delta$ cannot be resolved. In the current simulation, only the ${ }^{3} P_{J}$ Rydberg state is coupled to the ${ }^{3} S_{1}$ state. By including more states, the simulated trap loss might be enhanced and approach the measured values.

\section{CONCLUSIONS}

The present work demonstrates that even weak Rydberg dressing of a cold gas or BEC contained in an ODT can result in a rapid decrease in the ground-state atom population in the trap. One mechanism that can contribute to this decrease is transitions to neighboring Rydberg $P$ states driven by blackbody radiation, which results in strong interactions that broaden both the excitation and atom loss spectra. This limits the strength of the dressing that can be applied to a cold atom sample and for how long, suggesting that small sample sizes and/or short observation times may be essential for observing effects due to dressing [22,23]. Decoherence is also an important factor in trap loss, but its source remains to be clarified. However, it is interesting to note that studies of the effects of Rydberg interactions on the Autler-Townes spectra of ultracold strontium gases have revealed strong dephasing that dramatically alters the excitation dynamics and that results in a total loss of coherence of the dressed state that underlies the Autler-Townes structure at later times [12]. Experiments with dressed alkali atoms have revealed unexpectedly large interaction-induced broadening, which was also explained in terms of dipole-dipole interactions with neighboring $P$ states [24].

\section{ACKNOWLEDGMENTS}

We thank S. L. Rolston, J. V. Porto, and E. A. Goldschmidt for stimulating discussions on possible sources of enhanced loss in these experiments. Research was supported by the AFOSR under Grant No. FA 9550-12-1-0267, the NSF under Grants No. 1301773 and No. 1205946, the Robert A. Welch Foundation under Grants No. C-0734 and No. C-1844, the FWF (Austria) under Grant No. P23359-N16, and the SFBNextlite. The Vienna Scientific Cluster was used for the calculations.
[1] T. Pohl, E. Demler, and M. D. Lukin, Phys. Rev. Lett. 104, 043002 (2010).

[2] G. Pupillo, A. Micheli, M. Boninsegni, I. Lesanovsky, and P. Zoller, Phys. Rev. Lett. 104, 223002 (2010).

[3] R. M. W. van Bijnen, S. Smit, K. A. H. van Leeuwen, E. J. D. Vredenbregt, and S. J. J. M. F. Kokkelmans, J. Phys. B: Atom. Molec. Opt. Phys. 44, 184008 (2011).

[4] P. Schau, M. Cheneau, M. Endres, T. Fukuhara, S. Hild, A. Omran, T. Pohl, C. Gross, S. Kuhr, and I. Bloch, Nature (London) 491, 87 (2012).

[5] N. Henkel, R. Nath, and T. Pohl, Phys. Rev. Lett. 104, 195302 (2010).

[6] F. Maucher, N. Henkel, M. Saffman, W. Królikowski, S. Skupin, and T. Pohl, Phys. Rev. Lett. 106, 170401 (2011).
[7] M. Boninsegni and N. V. Prokof'ev, Rev. Mod. Phys. 84, 759 (2012).

[8] F. Cinti, P. Jain, M. Boninsegni, A. Micheli, P. Zoller, and G. Pupillo, Phys. Rev. Lett. 105, 135301 (2010).

[9] J. E. Johnson and S. L. Rolston, Phys. Rev. A 82, 033412 (2010).

[10] J. B. Balewski, A. T. Krupp, A. Gaj, S. Hofferberth, R. Löw, and T. Pfau, New J. Phys. 16, 063012 (2014).

[11] S. Helmrich, A. Arias, N. Pehoviak, and S. Whitlock, J. Phys. B 49, 03LT02 (2016).

[12] B. J. DeSalvo, J. A. Aman, C. Gaul, T. Pohl, S. Yoshida, J. Burgdörfer, K. R. A. Hazzard, F. B. Dunning, and T. C. Killian, Phys. Rev. A 93, 022709 (2016). 
[13] Y. N. Martinez de Escobar, P. G. Mickelson, M. Yan, B. J. DeSalvo, S. B. Nagel, and T. C. Killian, Phys. Rev. Lett. 103, 200402 (2009).

[14] S. Stellmer, F. Schreck, and T. C. Killian, in Annual Review of Cold Atoms and Molecules, edited by K. W. Madison, K. Bongs, L. D. Carr, A. M. Rey, and H. Zhai (World Scientific, Singapore, 2014).

[15] S. Kunze, R. Hohmann, H.-J. Kluge, J. Lantzsch, L. Monz, J. Stenner, K. Stratmann, K. Wendt, and K. Zimmer, Z. Phys. D Atoms Mol. Clusters 27, 111 (1993).

[16] B. J. DeSalvo, J. A. Aman, F. B. Dunning, T. C. Killian, H. R. Sadeghpour, S. Yoshida, and J. Burgdörfer, Phys. Rev. A 92, 031403 (2015).

[17] H. Schempp, G. Günter, M. Robert-de Saint-Vincent, C. S. Hofmann, D. Breyel, A. Komnik, D. W. Schönleber, M. Gärttner, J. Evers, S. Whitlock et al., Phys. Rev. Lett. 112, 013002 (2014).
[18] N. Malossi, M. M. Valado, S. Scotto, O. Morsch, E. Arimondo, and D. Ciampini, J. Phys.: Conf. Ser. 497, 012031 (2014).

[19] D. W. Schönleber, M. Gärttner, and J. Evers, Phys. Rev. A 89, 033421 (2014).

[20] A. Chotia, M. Viteau, T. Vogt, D. Comparat, and P. Pillet, New. J. Phys. 10, 045031 (2008).

[21] K. Mølmer, Y. Castin, and J. Dalibard, J. Opt. Soc. Am. B 10, 524 (1993).

[22] Y.-Y. Jau, A. M. Hankin, T. Keating, I. H. Deutsch, and G. W. Biedermann, Nat. Phys. 12, 71 (2016).

[23] J. Zeiher, P. Schauß, S. Hild, T. Macrì, I. Bloch, and C. Gross, Phys. Rev. X 5, 031015 (2015).

[24] E. A. Goldschmidt, T. Boulier, R. C. Brown, S. B. Koller, J. T. Young, A. V. Gorshkov, S. L. Rolston, and J. V. Porto, Phys. Rev. Lett. 116, 113001 (2016). 